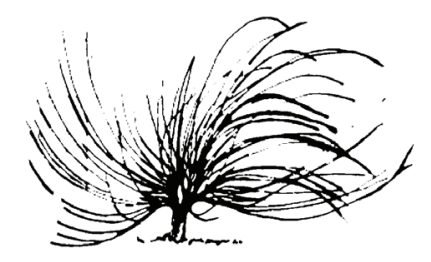

\title{
El aprendizaje por indagación como opción para desarrollar la unidad de hidrostática del programa de física de décimo año, de la Educación Diversificada de Costa Rica
}

\author{
Huberth Andrés Pérez-Villalobos ${ }^{1}$ \\ Universidad Nacional, \\ Heredia, Costa Rica \\ huberth16@gmail.com
}

\author{
María Isabel Torres-Salas ${ }^{2}$ \\ Universidad Nacional, \\ Heredia, Costa Rica \\ isabeltorresr@yahoo.com \\ Alexander Gómez-Lépiz ${ }^{3}$ \\ Universidad Nacional, \\ Heredia, Costa Rica \\ gmanatusl@gmail.com
}

\begin{abstract}
Resumen
La utilización del aprendizaje por indagación para abordar los temas de la unidad de hidrostática del programa de física de décimo año de la educación diversificada de Costa Rica es una opción metodológica que ayudaría
\end{abstract}

\section{(c) (1) @ (9)}

Recibido: 8 de noviembre de 2016-Aprobado: 3 de octubre de 2017

http://dx.doi.org/10.15359/rep.12-2.8

1 Profesor de cursos de Física, obtuvo su grado de licenciatura con honores. Departamento de Física, Facultad de Ciencias Exactas y Naturales, Universidad Nacional, Costa Rica

2 Profesora de cursos de educología, División de Educología, del Centro de Investigación en Educación, obtuvo su grado de maestría. Universidad Latina, Costa Rica

3 Profesor de cursos de Biología, obtuvo su grado de maestría. Escuela de Ciencias Biológicas, Facultad de Ciencias Exactas y Naturales, Universidad Nacional, Costa Rica. 
en la comprensión de estos temas, tal como se denota en la investigación que dio origen a este artículo, la cual trata sobre las estrategias metodológicas empleadas por los docentes al desarrollar los temas de hidrostática y las dificultades que presentan los estudiantes. Esta se realizó según el paradigma naturalista con un enfoque cualitativo y el tipo de estudio utilizado fue el "estudio de comunidad". Entre los principales hallazgos se encontró que la mayoría de los estudiantes tienen dificultades para comprender esa materia y que las estrategias metodológicas más utilizadas por los docentes provienen del modelo conductista, siendo la resolución de ejercicios en la pizarra la más usada. Además, los docentes tienen noción sobre qué es aprendizaje por indagación y son conscientes de las ventajas que ofrece el empleo de estrategias basadas en esta metodología, pero no la aplican porque no cuentan con estrategias metodológicas derivadas de secuencias didácticas en el marco de la enseñanza por indagación en este tema. Por último, cabe mencionar que el artículo proporciona tres actividades mediadas a través del aprendizaje por indagación para aplicar en la clase de física.

Palabras clave: física, educación, enseñanza de ciencias fundamentales, mecánica de fluidos y enseñanza de la física.

\begin{abstract}
The use of inquiry-based learning to address the topics of the hydrostatic unit of the 10th grade physics program of Costa Rican Diversified Education is a methodological option that would help in the understanding of these issues, as was denoted in the research, which gave rise to this article, dealing with the methodological strategies employed by teachers in developing hydrostatic topics and the difficulties presented by students. The research project conducted was developed under the naturalistic paradigm with a qualitative approach and the type of study used was the "community study". Among the main findings, it was discovered that the majority of students have difficulty understanding this subject and that the methodological strategies most commonly used
\end{abstract}


by teachers come from the behavioral model, being the resolution of exercises on the board the most used. In addition, teachers have a notion about learning by inquiry and are aware of the advantages offered by the use of strategies based on this methodology, but they do not apply it because they do not have methodological strategies derived from didactic sequences in the context of teaching by inquiry into this topic. Finally, it should be mentioned that this article provides three activities mediated through inquiry learning to apply them in the physics class.

Keywords: physics, education, basic science education, fluid mechanics, physics teaching.

\section{Introducción}

El ser humano siempre ha tenido curiosidad por el mundo que le rodea y también por cómo funciona ese mundo. Cuando se aprende mediante la indagación, los estudiantes están buscando soluciones y haciendo nuevas preguntas, porque se involucran activamente en hacer observaciones, recolectar, analizar y sintetizar información, sacar conclusiones y desarrollar habilidades que les serán útiles para resolver problemas. Además "las actividades orientadas a la indagación, incrementan la propia comprensión de los temas y procedimientos que resultan importantes porque contribuyen a desarrollar aquello que la persona necesita saber y dominar" (Whells y Mejia, 2005, p. 3).

En esta misma línea, estas habilidades pueden ser aplicadas en la enseñanza y aprendizaje de las diferentes materias del currículum, ayudando a que las personas tengan una mayor comprensión y, por ende, un mayor aprendizaje de los distintos temas que se desarrollan a lo largo de su vida como estudiantes en los diferentes niveles de la educación formal.

En este sentido la física no es la excepción, al aplicar la enseñanza por indagación en la física se estará apoyando el pensamiento de los alumnos y, a la vez, incentivando el desarrollo de sus mentes para lograr nuevos aprendizajes de manera creativa; de esta forma los discentes estarán aprendiendo mediante la comprensión de cómo abarcar y darles sentido a los diversos tópicos de esta disciplina científica. 
Sin embargo, en lo que respecta a la enseñanza de esta disciplina, existen dificultades de comprensión de esta y, además, parece no contar con el aprecio de la mayoría de los estudiantes que cursan la educación diversificada, como lo muestran las estadísticas presentadas en la tabla 1 que revelan la poca escogencia de esta ciencia por parte de los estudiantes a la hora de presentar el examen de bachillerato en Costa Rica.

Tabla 1. Número de estudiantes que seleccionaron física a nivel nacional en el periodo 2008-2013

\begin{tabular}{|c|c|c|c|}
\hline Año & $\begin{array}{c}\mathbf{N}^{\circ} \text { de estudiantes } \\
\text { que presentaron } \\
\text { pruebas de } \\
\text { bachillerato }\end{array}$ & $\begin{array}{c}\mathbf{N}^{\circ} \text { de estudiantes } \\
\text { que presentaron } \\
\text { la prueba de física }\end{array}$ & Porcentaje (\%) \\
\hline 2008 & 32551 & 3619 & 11,10 \\
\hline 2009 & 23682 & 2731 & 11,50 \\
\hline 2010 & 23126 & 2272 & 9,80 \\
\hline 2011 & 35467 & 3088 & 8,71 \\
\hline 2012 & 35033 & 3029 & 8,65 \\
\hline 2013 & 36130 & 3249 & 8,99 \\
\hline 2014 & 38340 & 3524 & 9,19 \\
\hline
\end{tabular}

Fuente: Resultados de las pruebas nacionales de la educación formal de cada año. Dirección de Gestión y Calidad (MEP, 2008-2015).

Lo anterior ocurre a pesar de que la física es una ciencia que se considera la base fundamental de otras ramas del saber como la meteorología, la astronomía, la geología y las ingenierías, las ciencias biológicas, biomédicas, y además, ayuda a comprender muchos de los fenómenos de la vida cotidiana desde el rayo que cae en una tormenta eléctrica, hasta el funcionamiento de un horno microondas o un teléfono celular. Sin embargo, esta ciencia parece invisibilizada y sentenciada a ser impartida en las aulas escolares como una asignatura compleja y llena de fórmulas matemáticas, en parte porque los métodos utilizados para enseñarla en la mayoría de los casos han estado desligados de la realidad, ocasionando la no comprensión de esta como acota Sagan (1989), citado por Padrón (2012): "Vivimos en una sociedad profundamente dependiente de la ciencia y la tecnología, en la que nadie sabe nada de estos temas. Ello constituye una fórmula segura para el desastre" (p. 27). 
En esta misma línea Barahona y Ramírez (2011), como resultado de su investigación encontraron que en la mayoría de las clases de física los docentes utilizaban la resolución de ejercicios de una manera mecánica y sus resultados no los relacionaban con la vida cotidiana. También resaltaron que los estudiantes mostraban gran desinterés en las clases aduciendo que la física no era una materia de su agrado.

Rojas (2006) afirma que el conductismo tradicional con el cual las lecciones de física se han desarrollado a lo largo de este tiempo, no ha dado los frutos que se esperaban, ya que la educación tradicional tiene muchas limitantes como la de no promover que el estudiante construya su propio conocimiento y relacione lo aprendido con lo que le rodea. Por ello, este autor también indica que es necesario implementar nuevas estrategias metodológicas en el proceso de enseñanza-aprendizaje, que hagan que el estudiante se apropie del conocimiento.

En este sentido, en Argentina Bizzio et al. (2011) buscaron cuál es el impacto que causan las estrategias cognitivas y metacognitivas en la enseñanza de las ciencias naturales. La investigación reveló un impacto muy positivo, ya que el uso de estas estrategias metodológicas logró que el alumnado asumiera un rol activo en la lectura, que tomara conciencia de su nivel de comprensión del texto y adquiriera de manera significativa los conocimientos impartidos en clase.

Dado que diferentes estudios como los mencionados apuntan a la necesidad de un cambio en las estrategias para la enseñanza de la física, esta investigación buscó proponer estrategias metodológicas basadas en el aprendizaje por indagación, porque según Harlen (2013) éste permite, que el estudiante encuentre relación entre la materia vista en clase y el mundo físico que experimenta todos los días, además permite que inicie su aprendizaje con la recolección de información a través de la aplicación de los sentidos humanos, lo que permitirá que los estudiantes generen preguntas, investiguen y realicen sus descubrimientos.

\section{Referentes teóricos}

La hidrostática es la rama de la mecánica que estudia todos los fluidos en estado de equilibrio, sin que aparezcan fuerzas que alteren su movimiento o posición, en otras palabras, en la hidrostática se estudian las interacciones de los fluidos en reposo con su interior y su entorno, a 
diferencia de la hidrodinámica que estudia las interacciones internas y externas de los fluidos en movimiento.

Como parte de esta rama de la física se estudian dos principios el de Pascal y el de Arquímedes. El principio de Pascal se define según Sears, Zemansky, Young y Freedman (2009) como: "la presión aplicada a un fluido encerrado se transmite sin disminución a todas las partes del fluido y las paredes del recipiente" (p. 460).

Por otra parte, el principio de Arquímedes enuncia que si un cuerpo esta parcial o totalmente sumergido en un fluido, este ejerce una fuerza hacia arriba sobre el mismo cuerpo de igual magnitud al peso del fluido desplazado por dicho cuerpo (Wilson, Buffa y Lou, 2007).

Estos principios mencionados forman parte del programa de física de la educación diversificada de Costa Rica y constituyen uno de los tópicos con los cuales los estudiantes presentan dificultades para su comprensión y contextualización (MEP, Dirección de Gestión y Calidad, 2008-2015), por lo que se considera de vital importancia investigar cómo se enseñan estos contenidos. Es sabido que la física por décadas se ha impartido bajo un modelo tradicional donde prevalece la transmisión de hechos, teorías científicas y aplicaciones tecnológicas, contribuyendo a que sea vista como una asignatura compleja y llena de fórmulas matemáticas, lo cual también queda evidente en recientes estudios sobre la percepción que existe sobre esta materia según lo señalan Katsampoxaki-Hodgetts et al. (2015).

Pero las nuevas tendencias didácticas ponen de manifiesto la importancia de contar con profesores que tengan una actitud reflexiva y crítica, que los lleve a tomar decisiones orientadas al diseño y la implementación de nuevas opciones que mejoren su práctica pedagógica; pero sobre todo dispuestos a motivar a los educandos para que el aprendizaje de esta disciplina se torne atractiva para ellos y le encuentren utilidad para resolver o entender problemas del entorno y situaciones de su diario vivir.

Por medio de la enseñanza de las ciencias y de la física en particular, se debe fomentar en los estudiantes un pensamiento científico y con ello formar un ciudadano con un pensamiento más crítico, con el fin de contribuir a la formación de una sociedad más alfabetizada científicamente. Sin embargo, la enseñanza de la física enfrenta muchos desafíos en la actualidad, uno de ellos y tal vez el que necesita más atención es ¿Por qué resulta difícil aprender física? Al respecto 
Pozo y Gómez (2009) intentan dar una respuesta, cuando plantean que la dificultad de aprendizaje tiene diferentes causas, entre ellas están las relacionadas con las características propias de la disciplina, la forma en que los alumnos aprenden y las metodologías empleadas por los docentes que en la mayoría de los casos están desvinculadas de la realidad. De ahí la importancia de enseñar con estrategias didácticas que no solo puedan atender las diferentes formas como aprenden los estudiantes, sino que además permitan contextualizar ese aprendizaje.

A pesar de los esfuerzos realizados en aras de cambiar la forma en la cual se enseña esta disciplina, pareciera que todavía se utilizan los mismos libros de texto y las mismas metodologías en su mayoría de corte conductista y academicista. Ante esto se ha visto la necesidad de impulsar otras formas de enseñar física, desde un paradigma cognoscitivo que concibe al estudiante como el actor principal del aprendizaje, donde no solo tiene importancia lo que se aprende y sus procedimientos sino la relación y utilidad de lo aprendido con el contexto inmediato.

Lo anterior está demostrado en la investigación de Barahona y Ramírez (2011) donde los resultados arrojaron que en la mayoría de las clases de física desarrolladas por los docentes, se evidenciaba que el profesor utilizaba la resolución de ejercicios. Sin embargo, estas resoluciones no estaban relacionadas con la vida cotidiana y provocaban una mecanización en los estudiantes. También resaltaron el hecho de que existe un gran desinterés por parte del alumno, donde se demostraba que la física no era una materia de agrado entre la población estudiantil.

\section{Aprendizaje por indagación}

En cuanto a la indagación se puede definir como un método caracterizado por la curiosidad y la investigación. Las personas hacen indagación desde su nacimiento hasta el momento de su muerte. El postulado: "Dímelo y se me olvidará, muéstramelo y lo recordaré, involúcrame y entenderé" es la esencia del aprendizaje por indagación (Escalante, 2008, p. 2). Lo anterior hace referencia a que la indagación es un proceso que el ser humano lo ha realizado desde siempre, por ello se podría decir que es casi una necesidad imperiosa desde que comienza su vida hasta que finaliza.

El ser humano siempre ha tenido curiosidad por el mundo que le rodea y también por cómo funciona y uno de los logros más relevantes 
de la indagación es permitir al alumno ese entendimiento del mundo y adquirir ese nuevo conocimiento, el cual le va a servir para su vida (Contreras, 2006). Otro logro de las estrategias metodológicas basadas en la indagación es que permiten a estudiantes y profesores tomar roles muy diferentes en comparación a los roles que se utilizan en la enseñanza tradicional. De acuerdo con Calderón, Hernández y Villalobos (2011) estos roles que los estudiantes -una vez conformados en grupos de cuatro personas pueden desempeñar son los siguientes: el encargado del material, el secretario, el director científico y el vocero. El hecho de que a los estudiantes que conforman un grupo se les asignen diferentes tareas, los hace copartícipes de su propio aprendizaje, ya que cada estudiante tiene un papel activo dentro del grupo.

Por otra parte, el rol del profesor también cambia porque debe ser un mediador y no un director del aprendizaje, promoviendo que los estudiantes se conviertan en miembros activos del proceso de enseñanza-aprendizaje y según Arenas (2005) para que la metodología indagatoria permita un adecuado proceso de enseñanza y aprendizaje, esta debe ser guiada y mediada por el docente en la sala de clases, trabajando las siguientes cuatro etapas:

- Focalización: en esta etapa se exploran y explicitan las ideas que traen los estudiantes con respecto a la temática, problema o pregunta a investigar.

- Exploración: se inicia con la discusión y realización de una experiencia cuidadosamente elegida, para que los estudiantes puedan comprobar si sus ideas acerca del tema o fenómeno en cuestión se ajustan a lo que ocurre en la realidad.

- Comparación o contraste: luego de realizada la experiencia, se confrontan las predicciones realizadas con los resultados obtenidos, para que los estudiantes elaboren sus propias conclusiones con respecto al problema analizado.

- Aplicación: el objetivo de esta es poner al estudiante ante nuevas situaciones que ayuden a afirmar el aprendizaje y asociarlo al acontecer cotidiano.

Según Escalante (2008), el aprendizaje por indagación origina un pensamiento crítico, desarrollando mayor habilidad en los procesos de las ciencias y como la capacidad para la resolución de problemas. 


\section{Metodología}

La investigación realizada se estructuró de la siguiente manera: Se efectuó un diagnóstico acerca de las estrategias metodológicas empleadas por los docentes al desarrollar los temas de la VII unidad de hidrostática del programa de física para décimo año y se identificaron las dificultades que presentan los estudiantes cuando se desarrolla la unidad mencionada. Además, se estimó conveniente conocer la opinión de los docentes acerca de la utilización del aprendizaje por indagación como estrategia metodológica en el desarrollo del tema de hidrostática, con el fin de proponer algunas secuencias didácticas en el marco de la enseñanza por indagación en este tema.

La investigación se realizó según el paradigma naturalista con un enfoque cualitativo y el tipo de estudio utilizado fue el "estudio de comunidad". Para ello se trabajó con una sección de décimo año de una institución académica en Heredia, Costa Rica, conformada por 25 estudiantes y el profesor que imparte la clase de Física. Además, participaron 9 profesores que también imparten física en el nivel de décimo en otras instituciones. La escogencia de la muestra fue aleatoria y el tamaño fue intencionado, ya que lo que se pretendió fue conocer a profundidad diferentes aspectos relacionados con la enseñanza del tema de hidrostática. Para obtener la información, se utilizaron diferentes instrumentos como entrevistas semiestructuradas, cuestionarios abiertos y registros anecdóticos para anotar la información obtenida durante las observaciones realizadas cuando se desarrollaron los temas referentes a hidrostática. Los instrumentos empleados fueron validados por criterios de expertos, tanto en el campo de la física como en el de pedagogía.

Los resultados y la discusión se realizaron considerando las siguientes categorías de análisis:

- "Estrategias metodológicas empleadas por los docentes". Donde se tomaron como subcategorías, las estrategias que utilizó el docente para dar la clase de física cuando se impartía el tema de hidrostática, los recursos empleados, así como el rol que ejerce $y$, por último, si relaciona los contenidos con situaciones reales.

- $\quad$ "Dificultades que presentan los estudiantes de décimo año cuando se desarrollan los temas de la VII unidad de hidrostática". Se consideraron como subcategorías, las dificultades que presentan 
en el aprendizaje de esos temas, la opinión acerca de las estrategias utilizadas por el docente y las formas en que les gustaría que les enseñaran estos temas.

- $\quad$ "Percepción de los profesores de física acerca de estrategias metodológicas basadas en la indagación para la enseñanza de la física". Se tomó en cuenta la opinión que tienen los profesores sobre las ventajas de la aplicación de la enseñanza por indagación y su disposición para enseñar los temas utilizando estrategias metodológicas basadas en la indagación.

\section{Resultados y discusión}

\section{Estrategias metodológicas empleadas por los docentes}

Se determinó que el docente imparte su clase de una manera expositiva y los estudiantes resuelven ejercicios siguiendo instrucciones muy precisas. Este es un método asociado con un modelo tradicionalista que tiene como base el conductismo, ya que los estudiantes realizan los ejercicios en la mayoría de los casos aplicando fórmulas mediante métodos transmitidos por el profesor. Según Quinquer (2004), la clase expositiva debilita el desarrollo de habilidades como buscar, seleccionar, organizar, presentar información, trabajar en equipo, afrontar, resolver problemas reales, aplicar técnicas y desarrollar un pensamiento crítico. De ahí que este tipo de clase en las que utilizan con frecuencia las metodologías tradicionales, no contribuyen significativamente al desarrollo de habilidades cognitivas, ya que por su naturaleza obligan al estudiante a ser un actor pasivo del proceso de enseñanza-aprendizaje.

Por otra parte, un porcentaje alto de estudiantes (84\%) califican de regular a mala las estrategias utilizadas por el profesor y el $80 \%$ dice que la resolución de ejercicios en la pizarra es la estrategia con la que más se trabaja en la clase (tabla 2), aspecto que es preocupante, ya que existe una amplia gama de estrategias que permiten al estudiante tener un papel más activo en la construcción del conocimiento. Es aquí donde precisamente deben tenerse en cuenta las nuevas metodologías de aprendizaje donde se pretende que el alumno sea un protagonista del proceso de aprendizaje y el profesor un mediador del mismo (Donaire, Gallardo, y Marcia, 2006). 
Tabla 2. Percepción de los estudiantes acerca de las estrategias metodológicas, que utiliza el docente en la clase al desarrollar los temas de hidrostática

\begin{tabular}{|l|l|c|c|}
\hline \multicolumn{1}{|c|}{ Aspecto } & Respuesta de los estudiantes & F & (\%) \\
\hline $\begin{array}{l}\text { 1. ¿Cómo califica las estrategias } \\
\text { que utiliza el profesor } \\
\text { para desarrollar el tema de } \\
\text { hidrostática? }\end{array}$ & Buenas & 4 & 16 \\
\cline { 2 - 4 } & Regulares & 15 & 60 \\
\cline { 2 - 4 } $\begin{array}{l}\text { 2. Estrategias que el profesor } \\
\text { utiliza con más frecuencia al } \\
\text { impartir la clase de física. }\end{array}$ & $\begin{array}{l}\text { Mesolución de ejercicios en } \\
\text { la pizarra }\end{array}$ & 20 & 80 \\
\cline { 2 - 4 } & Demostraciones & 5 & 20 \\
\hline
\end{tabular}

Fuente: Propia de la investigación (F: frecuencia).

Por otra parte, en esta investigación como parte de las observaciones realizadas, se evidenció que muy pocas veces el docente desarrolló actividades que permitieran hacer un enlace de la materia vista en clase con la vida cotidiana. Sobre este aspecto también los estudiantes señalan que el docente imparte la clase enfatizando que los contenidos desarrollados se deben aprender para el examen, sin ligarlos de alguna forma con su vida diaria. Sin embargo, ellos expresan que les gustaría que la física se les vinculara con aplicaciones reales del entorno. Por ello es importante pensar en opciones como el aprendizaje por indagación, que propone la necesidad de investigar el entorno y contrastar los saberes aprendidos (Arenas, 2005). Esto evidencia que existen opciones metodológicas que promueven el que se vincule la materia vista en la clase con el medio que los rodea.

Acerca de los recursos didácticos utilizados por los docentes se constató que emplean principalmente la pizarra y el libro de texto, aspecto que también lo confirman los estudiantes, al mencionar estos recursos como los más empleados. La pizarra no es un recurso didáctico malo, incluso es uno de los más usados e importantes en la enseñanza de la física, pero el abuso limita la utilización de otros recursos que pueden contribuir a facilitar su aprendizaje. En relación con la selección de recursos didácticos que hace un docente a la hora de planear la clase, Fonseca (2006) expresa que es un aspecto muy importante, ya que éstos deben constituir herramientas fundamentales para el desarrollo y enriquecimiento del proceso de enseñanza-aprendizaje de los alumnos. 
También se percibió, que el rol asumido por el docente durante la clase era de "director" del proceso de aprendizaje, además, los estudiantes afirman que ven al profesor como aquel que dirige su proceso de aprendizaje, lo que confirma lo observado. Este desempeño del docente como director del proceso es típico de un modelo conductista y si bien es cierto, que en algunos momentos permite que los estudiantes le hagan consultas, él las contesta de forma directa y no interactiva, por ello los estudiantes prácticamente no intervienen en la construcción de su aprendizaje, lo anterior se contrapone a las nuevas tendencias constructivistas. En este mismo sentido, Ñeco (2005) recomienda, que el profesor sea un mediador del aprendizaje, para que catalice el aprendizaje de sus alumnos.

En concordancia con lo anterior, Gómez (2012) afirma que entre los elementos que pueden contribuir a que el docente tenga un papel distinto al tradicional en sus clases, está la utilización de metodologías que conlleven por su propia dinámica a este cambio en el rol del profesor. Por ello la indagación es una de las metodologías que puede propiciar este cambio, ya que en el marco de este tipo de aprendizaje el docente desarrolla el rol de mediador del proceso de enseñanza y aprendizaje, por lo que deja de ser aquel que todo lo sabe y todo lo responde y pasa a ser más bien, el arquitecto del proceso indagatorio, asignando papeles y velando porque todos cumplan su parte. Según Calderón, Hernández y Villalobos (2011) la indagación permite que el docente desarrolle el proceso siendo un mediador de manera que los estudiantes vayan descubriendo los conceptos y adquiriendo habilidades que les servirán para enfrentar la vida.

\section{Dificultades que presentan los estudiantes de décimo año cuando se desarrollan los temas de la VII unidad de hidrostática}

Se determinó que, al avanzar el profesor en el desarrollo de esta unidad, los estudiantes van teniendo más problemas con los conceptos, las fórmulas y los cálculos. También ellos opinaron que tienen dificultades con esos conceptos durante el desarrollo de las clases (tabla 3) e indicaron los temas que más se les dificultó, además expresan que el profesor desarrolla la materia de manera muy rápida, lo que dificulta más el entendimiento de la teoría. 
Tabla 3. Percepción de los estudiantes acerca de las dificultades para entender los temas de hidrostática

\begin{tabular}{|c|c|c|}
\hline Aspecto & Respuesta de los estudiantes & $\mathbf{F}$ \\
\hline \multirow{3}{*}{$\begin{array}{l}\text { Dificultades que se le presentaron } \\
\text { a usted cuando se desarrolló en } \\
\text { clase los temas de hidrostática }\end{array}$} & $\begin{array}{l}\text {-El despeje de fórmulas y } \\
\text { en general los temas se nos } \\
\text { dificultan mucho }\end{array}$ & 10 \\
\hline & -Entender las demostraciones & 8 \\
\hline & -Hacer los ejercicios & 7 \\
\hline \multirow{4}{*}{$\begin{array}{l}\text { Temas de hidrostática que les } \\
\text { resultaron más difíciles de } \\
\text { comprender a los estudiantes y } \\
\text { por qué }\end{array}$} & -Presión y densidad & 9 \\
\hline & -Presión atmosférica & 9 \\
\hline & -Ley de Boyle & 5 \\
\hline & -Principio de Arquímedes & 2 \\
\hline
\end{tabular}

Fuente: Propia de la investigación (F: frecuencia).

Existen muchos factores que intervienen en el momento del aprendizaje de un determinado tema, pero sin duda las estrategias y los recursos que utiliza el docente tienen mucha influencia en la forma como los estudiantes abordan el proceso de aprendizaje en la clase, y cuando estas estrategias metodológicas son de corte conductista en la mayoría de los casos están basadas en la transmisión y memorización sin que exista una comprensión real de lo que están "aprendiendo". Un aspecto importante de resaltar es que el programa de estudio propone que se realicen demostraciones y experimentaciones sobre el tema, sin embargo, en la práctica docente no se presentan. Lo anterior lo afirma el programa de educación diversificada en el apartado de "orientaciones generales para la mediación docente", cuando menciona que "Un método activo propicia vivir y clarificar las actividades propias del quehacer científico. Como hacer uso de "lluvia de ideas", mesas redondas, demostraciones, prácticas de laboratorio, guías y visitas de estudio, lecciones prácticas, análisis de temas de interés para el alumno, discusión de tópicos de actualidad, entre otras".

Esto evidencia la necesidad de implementar estrategias metodológicas, que le permitan un rol más participativo al estudiante y que considere los saberes previos que estos traen, un aspecto necesario para desarrollar una base conceptual sólida y desarrollar un pensamiento crítico (Villarreal et al., 2007). 
En esta misma línea, las estrategias didácticas fundamentadas en el aprendizaje por indagación se perfilan como una excelente opción para mediar el conocimiento en las aulas de los centros educativos. Según Escalante (2008), la indagación facilita la comprensión de lo que se está enseñando, ayuda a contextualizar los saberes y a establecer conexiones con el medio que los rodea, aspecto que contribuye a que lo aprendido tenga significado; por ello debe usarse como una estrategia en el proceso de enseñanza y aprendizaje. De ahí que se recomiende como una alternativa para que los profesores de física la utilicen en el desarrollo de sus lecciones.

\section{Percepción de los profesores de física acerca de estrategias metodo- lógicas basadas en la indagación para la enseñanza de la física}

La mitad de los educadores de la muestra indican que el uso de la indagación como estrategia metodológica ayuda a que los estudiantes se apropien del conocimiento, porque este aprendizaje permite que el estudiante descubra el mundo a su alrededor. Además, la otra mitad tiene concepciones similares, como se puede ver en la tabla 4 .

Los docentes comprenden que mediante la indagación los estudiantes reestructuran el conocimiento previo que traen y obtienen conclusiones del mundo que los rodea. Lo que indica que, aparte de tener noción de esta estrategia metodológica de aprendizaje, también conocen las ventajas que le puede traer a la clase el empleo de esta.

Tabla 4. Opinión de los docentes de su percepción sobre la enseñanza por indagación como estrategia metodológica

\begin{tabular}{|l|l|c|}
\hline \multicolumn{1}{|c|}{ Aspecto } & \multicolumn{1}{|c|}{ Respuesta de los docentes } & F \\
\hline $\begin{array}{l}\text { Ventajas que los docentes } \\
\text { consideran que traería el } \\
\text { aprendizaje por indagación } \\
\text { como estrategia metodológica } \\
\text { para sus clases. }\end{array}$ & $\begin{array}{l}\text { Que se apropian del conocimiento, } \\
\text { porque en la indagación el estudiante } \\
\text { descubre un mundo a su alrededor. }\end{array}$ & 5 \\
\cline { 2 - 3 } & $\begin{array}{l}\text { Mediante la indagación los } \\
\text { estudiantes reestructuran el } \\
\text { conocimiento previo que traen. }\end{array}$ & 3 \\
\cline { 2 - 3 } & $\begin{array}{l}\text { Mediante la indagación los } \\
\text { estudiantes obtienen conclusiones } \\
\text { del mundo que les rodea. }\end{array}$ & 2 \\
\hline
\end{tabular}

Fuente: Propia de la investigación (F: frecuencia). 
Los datos contenidos en la tabla 4 reafirman que los profesores saben que esta metodología conduce al estudiante a la construcción de su conocimiento relacionándolo además con el entorno en el que está inmerso; tal como lo describe Arenas (2005),

...con el modelo indagatorio, los estudiantes podrán apropiarse no sólo de los contenidos sino, además, de los procesos, en ese sentido, una de sus características más notables es que está orientado a superar uno de los problemas más frecuentes en la enseñanza tradicional de las ciencias en el aula: la tendencia a ofrecer respuestas a preguntas que los jóvenes nunca se han planteado (p. 1).

Además, en la entrevista todos los profesores opinan que estarían dispuestos a usar la estrategia metodológica del aprendizaje por indagación y admiten que la estrategia metodológica que usan actualmente no les permite a los estudiantes relacionar la materia vista en clase con la vida cotidiana. También reconocen la importancia de la indagación como estrategia metodológica para desarrollar sus clases, ya que esta lograría que los estudiantes comprendan de manera más fácil los contenidos desarrollados y que logren relacionar la materia vista en clase con los hechos que experimentan cada día como parte del entorno inmediato.

Con el fin de aplicar en temas de hidrostática la estrategia metodológica del aprendizaje por indagación se presentan a continuación tres ejemplos con actividades que responden a esta metodología.

\section{Indicaciones generales para todas las actividades}

* Formación de grupos: El profesor organiza a los estudiantes en grupos de cuatro personas y les asigna los roles. Como parte de la aplicación de la metodología por indagación es necesario que los grupos de trabajo estén conformados por cuatro personas a las que se les asignan roles claramente establecidos, con el fin de que cada miembro tenga una participación activa en el proceso: el $\mathrm{N}^{\circ} 1$. Encargado del material: Recoge el material, lo cuida, deja que todos lo manipulen, el $\mathrm{N}^{\circ}$ 2. Secretario: Hace dibujos, escríbe la hoja de informe de grupo de forma clara, recopila las ideas de todos de forma concisa, el $\mathrm{N}^{\circ} 3$. Director científico: Se encarga de que todos participen, se asegura de que todos siguen las instrucciones y es el encargado del tiempo, el $\mathrm{N}^{\circ} 4$. Vocero: Expone las conclusiones al grupo. 
** Dinámica de la actividad: La cual consiste en responder una interrogante que el profesor va a presentar y se preparara una respuesta que luego se expondrá a la clase (el profesor en todo momento debe procurar actuar como mediador del proceso educativo y no dirigirlo directamente).

*** Exposición: El docente solicita al vocero de cada grupo que pase en orden al frente de los demás grupos y exponga su respuesta a la interrogante planteada al inicio de la actividad.

\begin{tabular}{|c|c|}
\hline \multicolumn{2}{|c|}{ Título de la actividad I: La verdadera forma de los líquidos } \\
\hline \multicolumn{2}{|c|}{$\begin{array}{l}\text { Objetivo } \\
\text { Lograr la asimilación del concepto de fluidos, así como la comprensión de } \\
\text { la forma de un líquido sin el efecto de la gravedad en estudiantes de décimo } \\
\text { año, utilizando como estrategia metodológica el aprendizaje por indagación. } \\
\text { Materiales: Agua, alcohol etílico, aceite, jeringa con aguja, beacker o vaso } \\
\text { de forma similar. }\end{array}$} \\
\hline \multicolumn{2}{|c|}{$\begin{array}{l}\text { A continuación, se describen las fases que comprende el ejercicio basado en } \\
\text { las metodologías de la indagación. }\end{array}$} \\
\hline \multicolumn{2}{|c|}{ 1. Formación de grupos* } \\
\hline \multicolumn{2}{|c|}{ 2. Dinámica de la actividad** } \\
\hline Fase & Descripción \\
\hline 3a. Pregunta Inicial & $\begin{array}{l}\text { Una vez asignados los roles de cada grupo el } \\
\text { docente procede a realizar a los grupos la pregunta } \\
\text { indagatoria mediante el siguiente enunciado: } \\
\text { "Cuando vemos a los astronautas en la estación } \\
\text { espacial por medio de la televisión, observamos que } \\
\text { algunas veces sueltan gotas de agua en el medio } \\
\text { y estas toman forma de esfera y no se derraman, } \\
\text { entonces se nos puede hacer una contradicción, ya } \\
\text { que en la escuela nos decían que "los líquidos toman } \\
\text { la forma del recipiente que lo contiene...", nos nace } \\
\text { la pregunta, ¿cuáles son los factores que influyen en } \\
\text { la forma de los líquidos?" }\end{array}$ \\
\hline 3b.Tema & Fluidos (líquidos) \\
\hline $\begin{array}{l}\text { 3c. Aspectos del } \\
\text { tema para resolver } \\
\text { la pregunta inicial }\end{array}$ & Tensión superficial / densidad. \\
\hline
\end{tabular}


El aprendizaje por indagación como opción para desarrollar la unidad de hidrostática del programa de física de décimo año, de la

\begin{tabular}{|l|l|}
\hline $\begin{array}{l}\text { 4. Predicciones } \\
\text { intentos de } \\
\text { respuesta }\end{array}$ & $\begin{array}{l}\text { El profesor pide a cada grupo reunido por aparte } \\
\text { que elaboren posibles respuestas con sus debidos } \\
\text { argumentos que dan razonamiento lógico a sus } \\
\text { correspondientes respuestas, las cuales el secretario } \\
\text { las anota en el cuaderno de trabajo. }\end{array}$ \\
\hline $\begin{array}{l}\text { 5. Exploración o } \\
\text { investigación }\end{array}$ & $\begin{array}{l}\text { El docente entrega al encargado de material de cada } \\
\text { grupo un envase, una jeringa con aguja, alcohol, } \\
\text { aceite y agua. El docente pide a los estudiantes que } \\
\text { en el envase que se les entregó preparen una mezcla } \\
\text { de agua con alcohol en igual proporción, luego se } \\
\text { les solicita que introduzcan la jeringa hasta que la } \\
\text { aguja alcance la fase donde se encuentra el agua, } \\
\text { seguidamente se les pide que liberen con sumo } \\
\text { cuidado el aceite dentro de la fase donde se encuentre } \\
\text { la mayor parte de agua y observe lo que sucede con } \\
\text { el aceite. } \\
\text { Nota: todo lo realizado en la fase de exploración fue } \\
\text { con el fin de eliminar el efecto de la gravedad por el } \\
\text { aceite por medio de las diferencias de densidades de } \\
\text { los tres líquidos. }\end{array}$ \\
\hline $\begin{array}{l}\text { A continuación, el docente solicita a los grupos de } \\
\text { trabajo que contrasten lo que habían pronosticado } \\
\text { en sus hipotéticas respuestas con la experimentación } \\
\text { que realizaron en la fase anterior. El docente entrega } \\
\text { una hoja con conceptos como tensión superficial y } \\
\text { la información de la densidad de los tres líquidos } \\
\text { involucrados en la fase de experimentación, } \\
\text { seguidamente pide a los estudiantes que la lean, } \\
\text { luego reflexionen y reformulen si fuese necesario las } \\
\text { hipótesis realizadas en el punto anterior. }\end{array}$ \\
\hline 6. Reflexión & $\begin{array}{l}\text { A manera de evaluar lo aprendido el docente hace un } \\
\text { debate con los estudiantes donde se hable sobre por } \\
\text { qué los líquidos en la tierra se adaptan a la forma del } \\
\text { recipiente, pero en el espacio no y qué diferencia hay } \\
\text { entre los líquidos y los gases. }\end{array}$ \\
\hline Exposición***
\end{tabular}


Título de la actividad II: ¿Dónde se hierve el agua más rápido: en la playa o en la montaña?

\section{Objetivo}

Conseguir que los estudiantes comprendan cómo se relaciona la presión atmosférica con la altura por medio del aprendizaje por indagación.

Materiales: Fuente de calor, botella de plástico, clavo, pinzas para sostener el clavo, agua, cinta adhesiva.

\section{Metodología}

Indicaciones: En el siguiente cuadro se describen las fases que comprende el ejercicio basado en las metodologías de la indagación.

\begin{tabular}{|c|c|}
\hline \multicolumn{2}{|c|}{ 1. Formación de grupos* } \\
\hline \multicolumn{2}{|c|}{ 2. Dinámica de la actividad** } \\
\hline Fase & Descripción \\
\hline 3a. Pregunta Inicial & $\begin{array}{l}\text { El docente presenta a los grupos la pregunta } \\
\text { indagatoria mediante el siguiente enunciado: “En } \\
\text { nuestras vacaciones se nos ocurre ir a dos lugares, } \\
\text { uno es a una playa y el otro es a una montaña, para } \\
\text { disfrutar de los dos lugares al máximo, pero notamos } \\
\text { que nuestra mamá no tardó el mismo tiempo en hervir } \\
\text { el agua para el café en la montaña que el que tardó en } \\
\text { la playa, entonces nos hacemos la pregunta, ¿de qué } \\
\text { manera influye la altura en la ebullición del agua?" }\end{array}$ \\
\hline 3b.Tema & Presión atmosférica en dependencia de la altura. \\
\hline $\begin{array}{l}\text { 3c. Aspectos del } \\
\text { tema para resolver } \\
\text { la pregunta inicial }\end{array}$ & Presión / altitud. \\
\hline $\begin{array}{l}\text { 4. Predicciones } \\
\text { o intentos de } \\
\text { respuesta }\end{array}$ & $\begin{array}{l}\text { Por planteada la pregunta indagatoria el profesor } \\
\text { pide a cada grupo que elabore posibles respuestas } \\
\text { con sus debidos argumentos que dan razonamiento } \\
\text { lógico a sus correspondientes respuestas, las cuales el } \\
\text { secretario las anota en el cuaderno de trabajo. }\end{array}$ \\
\hline
\end{tabular}


El aprendizaje por indagación como opción para desarrollar la unidad de hidrostática del programa de física de décimo año, de la

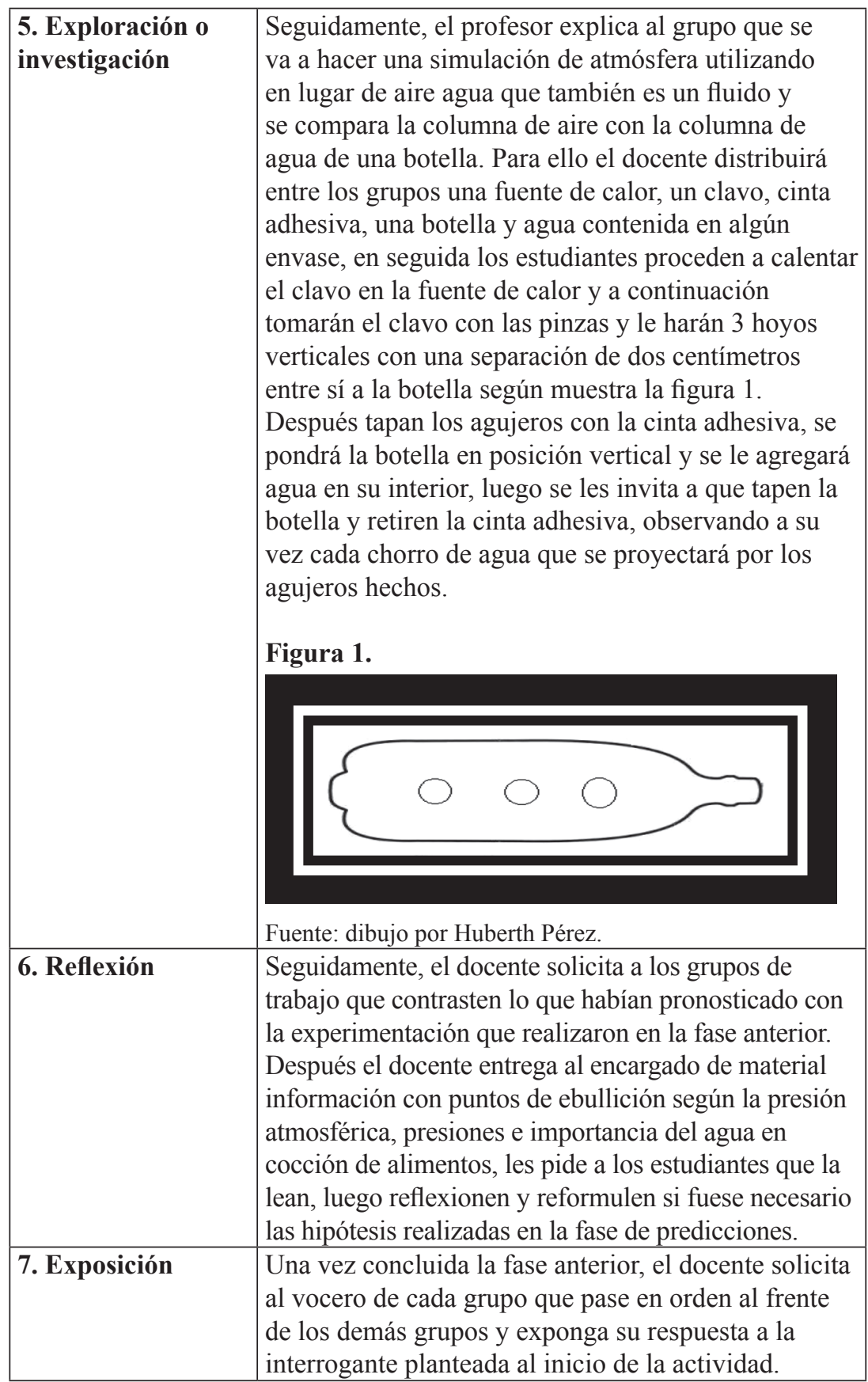




\begin{tabular}{|c|c|}
\hline $\begin{array}{l}\text { 8. Aplicación } \\
\text { (evaluación) }\end{array}$ & $\begin{array}{l}\text { A manera de evaluar lo aprendido el docente } \\
\text { procura hacer un debate donde los estudiantes den } \\
\text { una explicación al síndrome del buzo, el cambio } \\
\text { del punto de ebullición del agua y el problema de } \\
\text { ausencia de oxígeno que enfrentan los alpinistas en el } \\
\text { monte Everest. }\end{array}$ \\
\hline \multicolumn{2}{|c|}{ Título de la actividad III: Mis pulmones y Boyle } \\
\hline \multicolumn{2}{|c|}{$\begin{array}{l}\text { Objetivo } \\
\text { Lograr que los estudiantes relacionen la ley de Boyle y la función del } \\
\text { diafragma en el sistema respiratorio mediante el aprendizaje por indagación. } \\
\text { Materiales: Botella transparente con tapa, plastilina, alguna herramienta } \\
\text { para hacerle huecos a la tapa de la botella, dos globos, pajillas, guantes de } \\
\text { látex, cinta de embalar o similar }\end{array}$} \\
\hline \multicolumn{2}{|c|}{$\begin{array}{l}\text { Indicaciones: En el siguiente cuadro se describen las fases que comprende } \\
\text { el ejercicio basado en las metodologías de la indagación. }\end{array}$} \\
\hline \multicolumn{2}{|c|}{ 1. Formación de grupos* } \\
\hline \multicolumn{2}{|c|}{ 2. Dinámica de la actividad** } \\
\hline Fase & Descripción \\
\hline 3a. Pregunta inicial & $\begin{array}{l}\text { El docente presenta a los grupos la pregunta } \\
\text { indagatoria mediante el siguiente enunciado: } \\
\text { "Siempre que recibimos un mal golpe en la boca } \\
\text { del estómago se nos va el aire, esto debido a que el } \\
\text { diafragma sufre un espasmo y no podemos respirar } \\
\text { por más que queramos, entonces nos preguntamos, } \\
\text { ¿qué función cumple el diafragma en nuestro sistema } \\
\text { respiratorio? }\end{array}$ \\
\hline 3b. Tema & Ley de Boyle. \\
\hline $\begin{array}{l}\text { 3c. Aspectos del } \\
\text { tema para resolver } \\
\text { la pregunta inicial } \\
\end{array}$ & Presión / volumen / sistema respiratorio. \\
\hline $\begin{array}{l}\text { 4. Predicciones } \\
\text { o intentos de } \\
\text { respuesta }\end{array}$ & $\begin{array}{l}\text { Una vez planteada la pregunta indagatoria el profesor } \\
\text { solicita a los grupos que elaboren posibles respuestas, } \\
\text { las cuales el secretario las anotará en el cuaderno de } \\
\text { trabajo. }\end{array}$ \\
\hline
\end{tabular}


El aprendizaje por indagación como opción para desarrollar la unidad de hidrostática del programa de física de décimo año, de la

\begin{tabular}{|c|c|}
\hline $\begin{array}{l}\text { 5. Exploración o } \\
\text { investigación }\end{array}$ & $\begin{array}{l}\text { El docente explica a los grupos de trabajo que se va } \\
\text { a experimentar con un simulador de pulmones, para } \\
\text { ello distribuirá entre los grupos de trabajo, una botella } \\
\text { transparente, una herramienta para perforar, dos globos, } \\
\text { un guante de látex, dos pajillas, plastilina, cinta para } \\
\text { embalar y tijeras. Luego los estudiantes deben hacer } \\
\text { un corte transversal a la botella, seguidamente deben } \\
\text { colocar el guante de látex sobre el corte transversal y lo } \\
\text { asegura con la cinta de embalar, luego tomará la tapa } \\
\text { del mismo y le hace dos perforaciones suficientemente } \\
\text { grandes para que pasen las pajillas, además se toman } \\
\text { las pajillas y a cada una en uno de los extremos se le } \\
\text { pone el globo asegurándolo con la cinta de embalar, } \\
\text { por último se mete el extremo que no tiene globo de la } \\
\text { pajilla por la perforación de la tapa y se procede a sellar } \\
\text { el posible espacio entre la pajilla y el agujero, luego se } \\
\text { meten las pajillas con globos en la botella, enroscando } \\
\text { la pajilla. Al terminar los pasos indicados el sistema } \\
\text { debe verse como la figura } 2 \text {. A continuación se les pide } \\
\text { a los grupos de trabajo que tiren del guante (diafragma } \\
\text { artificial) y observen lo que sucede. } \\
\text { Figura } 2\end{array}$ \\
\hline 6. Reflexión & $\begin{array}{l}\text { A continuación el docente solicita a los grupos de } \\
\text { trabajo que contrasten lo que habían pronosticado } \\
\text { en sus hipotéticas respuestas con la experimentación } \\
\text { que realizaron en la fase anterior. Luego el docente } \\
\text { entrega un material que contenga la ley de Boyle, } \\
\text { información del diafragma y el sistema respiratorio, } \\
\text { seguidamente pide a los estudiantes que la lean, } \\
\text { luego reflexionen y reformulen si fuese necesario las } \\
\text { hipótesis realizadas en el punto anterior. }\end{array}$ \\
\hline
\end{tabular}




\begin{tabular}{|l|l|}
\hline 7. Exposición*** & \\
\hline $\begin{array}{l}\text { 8. Aplicación } \\
\text { (evaluación) }\end{array}$ & $\begin{array}{l}\text { A manera de evaluar lo aprendido el docente dejara } \\
\text { una tarea donde los estudiantes deben buscar } \\
\text { información y explicar desde el punto de vista de la } \\
\text { ley de Boyle por qué sucede el colapso pulmonar } \\
\text { cuando hay una perforación en el tórax especialmente } \\
\text { en la zona donde se encuentran los pulmones. }\end{array}$ \\
\hline
\end{tabular}

\section{Conclusiones}

En síntesis, se puede afirmar que la valoración realizada a través de la investigación permite sugerir que las técnicas didácticas actualmente utilizadas para el abordaje del tema relacionado con la hidrostática no favorecen el adecuado desarrollo de este, ya que estas en su mayoría son la resolución de problemas en la pizarra usando la exposición, metodología que está basada principalmente en el modelo conductista de la escuela tradicional, esto a pesar de las fuertes críticas que ha recibido dicho modelo en las últimas décadas. El cambio de estrategias de un enfoque de tipo conductista hacia un enfoque donde se utilicen metodologías de tipo constructivista, evita que los temas como la hidrostática sean enseñados de manera memorística, tal y como se percibe en los resultados planteados en el este estudio.

De lo anterior se puede afirmar que se evidencia la necesidad de acoger nuevas metodologías que le permitan al estudiante tener un papel protagónico en la apropiación del conocimiento, debido a que las metodologías tradicionales utilizadas por algunos profesores al enseñar los temas de hidrostática no contribuyen significativamente al desarrollo de habilidades cognitivas, ya que por su naturaleza obligan al estudiante a ser un actor pasivo del proceso de enseñanza-aprendizaje y terminan, en la mayoría de los casos, reduciendo este a una actividad memorística. Además, la mayor parte del tiempo no se relaciona la materia vista en clase con situaciones de la vida cotidiana.

Es decir, es necesario innovar e implementar otras formas diferentes al modelo tradicional para enseñar la física, que conduzcan a los estudiantes a tener aprendizajes significativos, y una alternativa podría ser la utilización de estrategias metodológicas basadas en la indagación donde pasos que la conforman permiten un abordaje óptimo, porque facilita el proceso de enseñanza-aprendizaje en temas relevantes 
y complejos de la física, ya que el enfoque constructivista, canalizado a través de este tipo de metodologías, permite que los estudiantes apliquen los conceptos aprendidos a la realidad de su entorno, lo cual facilitaría la comprensión de estos y su importancia en el diario vivir.

Por último, como parte del estudio realizado se evidenció que los profesores en su gran mayoría tienen una noción sobre qué es el aprendizaje por indagación y, a su vez, son conscientes de las ventajas que conlleva; además mostraron disponibilidad en utilizar la indagación como estrategia metodológica para enseñar la VII unidad de hidrostática. Por ello se plantearon algunas secuencias didácticas basadas en esta metodología y se le recomienda al Ministerio de Educación Pública que debería propiciar la capacitación del personal docente en metodologías de la indagación, ya que como se desprende de la opinión de los profesores de la muestra, ellos tienen el interés de aplicar nuevas metodologías para cubrir temas complejos como la hidrostática, sin embargo, requieren de capacitación adecuada en este tipo de iniciativas para un apropiado desarrollo de estas, que permita la mejora en la forma de impartir las lecciones y la comprensión de esos temas.

\section{Referencias}

Arenas, E. (2005). La indagación en la enseñanza y el aprendizaje de las ciencias. Recuperado de http://www.medellin.edu.co/sites/ Educativo/repositorio de recursos/La indagación en la enseñanza y el aprendizaje de las ciencias.pdf

Barahona, O. y Ramírez, J. (2011). Elaboración de un sitio web como propuesta metodológica para el proceso de enseñanza aprendizaje en las unidades de trabajo y energía del programa de física de educación diversificada (Tesis de licenciatura no publicada). Universidad Nacional de Costa Rica, Heredia.

Bizzio, M., Soliveres, M., Guirado, A. y Macías, A. (2011). Intervenciones didácticas en clases de ciencias naturales mediante el uso de estrategias cognitivas y metacognitivas de lectura. Revista Electrónica de la Enseñanza de las Ciencias, 10(3), 420-437. Recuperado de http://www.saum.uvigo.es/reec/volumenes/volumen10/REEC_10_3_2.pdf

Calderón, C., Hernández, C. y Villalobos, J. (2011). Curso de educación del pensamiento científico basado en la indagación articulado a 
los programas de estudio de ciencias en I y II ciclos. Recuperado en línea de http://www.mep.go.cr/sites/default/files/recursos/ archivo/modulo ciencias1.pdf

Contreras, F. (2006). Temas (2da. Ed.). Recuperado de http://www.eumed.net/libros-gratis/2007c/341/341.zip

Donaire, I., Gallardo, J. y Marcia, S. (2006). Nuevas metodologías en el aula. Revista Práctica docente, 1(3), 1-10. Recuperado de http:// www.cepgranada.org/ jmedina/articulos/n3_06/n3_06_57.pdf

Escalante, P. (2008). Aprendizaje por indagación. Recuperado de http:// www.medellin.edu.co/sites/Educativo/repositorio\%20de\%20recursos/Aprendizaje\%20por\%20indagaci\%C3\%B3n.pdf

Fonseca,M.(2006).Materialesyrecursosdidácticos, quéharíamossinellos. Recuperado de http://www.educaweb.com/noticia/2006/05/15/ materiales-recursos-didacticos-hariamos-ellos-1233/

Gómez, P. (2012). Método Moore o aprendizaje por indagación. Recuperado de http://www.webpgomez.com/index. php?option=com_content\&view $=$ article\&id=345\&Itemid $=189$

Harlen, W. (2013). Evaluación y Educación en Ciencias Basada en la Indagación: Aspectos de la Política y la Práctica. Publicado por Global Network of Science Academies (IAP) Science Education Programme (SEP). Recuperado de http://www.interacademies. net/File.aspx?id=22671

Katsampoxaki-Hodgetts, K., Fouskaki, M., Siakavara, K., Moschochoritou, R. y Chaniotakis, N. (2015). Student and Teacher Perceptions of Inquiry Based Science Education in Secondary Education in Greece. American Journal of Educational Research, 3(8), 968-976.

Ministerio de Educación Pública (MEP). (2008-2015). Dirección de Gestión y Calidad. Informes de bachillerato. Recuperado de $\mathrm{http}: / /$ www.dgec.mep.go.cr/deac/documentos

Neco, M. (2005). El rol del maestro en un esquema pedagógico constructivista. Recuperado de http://uocmaster-grupol.wikispaces. com/file/view/el_maestro_constructivista.pdf

Padrón, C.(2012). Administración, ciencia. Técnica y tecnología. Revista de Contaduría y Administración, 1(205), 27-35. Recuperado de revistas.unam.mx/index.php/rca/article/view/4539/4071

Perelman, Y.(2004). Física Recreativa. España: Grupo Anaya Comercial. 
Pozo, J. y Gómez, M. (2009). Aprender y enseñar ciencia. Madrid: Editorial Morata.

Quinquer, D. (2004). Estrategias metodológicas para enseñar y aprender ciencias sociales: interacción, cooperación y participación. Revista Iber, 40(1), 7-22.

Rojas, R. (2006). La creación de zonas de desarrollo próximo (ZPD) como estrategia de enseñanza aprendizaje constructivista en la resolución de problemas Lógico-Matemáticos, en una clase de física del ciclo de educación diversificada de una institución pública costarricense (Tesis de licenciatura no publicada). Universidad Nacional de Costa Rica, Heredia.

Sears, F., Zemansky, M., Young, H. y Freedman, R. (2009). Física Universitaria (décimo segunda edición). México: Editorial Pearson.

Villarreal, M., Lobo, H., Gutiérrez, G., Briceño, J., Rosario, J. y Díaz, J. (2007). La enseñanza de la física frente al nuevo milenio. Revista Academia, 4(8), 2-5. Recuperado de http://www.ecbichile.cl/ wp-content/uploads/2012/05/Aprendizaje-y-ensen\%CC\%83anza-de-ciencias-basados-en-la-indagacio\%CC\%81n..pdf

Whells, G. y Mejía, R. (2005). Hacia el diálogo en el salón de clases: enseñanza y aprendizaje por medio de la indagación. Revista Electrónica Sinéctica, 26(1), 1-19. Recuperado de http://www. redalyc.org/articulo.oa?id=99815914016

Wilson, J., Buffa, A. y Lou, B. (2007). Física. México: Editorial Pearson. 